\title{
Reseñas bibliográficas
}

Alejandro G. Vigo, Estudios aristotélicos, EUNSA, Pamplona, 2006, $475 \mathrm{pp}$.

El volumen compila catorce artículos publicados previamente en diversos libros y revistas. Se agrupan en tres partes: filosofía teórica, filosofía práctica y una pequeña sección dedicada a la discusión, donde se debate con los profesores Jonh J. Cleary y Niels Öffenberger. Esta última quizá hubiese cabido en la primera, según se verá más adelante.

Estudios aristotélicos, advierte su autor, no presenta unidad temática estricta y no debe considerarse una obra de tipo monográfico. En consecuencia, la mejor manera de hacerse una idea cabal del texto es reseñando cada uno de los capítulos por separado. No obstante, ciertas preocupaciones surcan el texto a todo lo largo. Los conceptos de prioridad, tiempo y verdad aparecen una y otra vez. De esta suerte, aunque el libro carece del formato de una monografía, sí presenta dos o tres hilos conductores que permiten una lectura continuada de los artículos del volumen.

La primera parte del libro se afana en demostrar la prioridad de la sustancia en el orden ontológico, lógico y gnoseológico. Repetidamente, Vigo subraya la necesidad de no perder de vista que tales órdenes se encuentran entrelazados en el corpus y que tal entramado es, ni más ni menos, que uno de los rasgos distintivos de la metafísica del Estagirita.

El capítulo I, "Prioridad y prioridad ontológica según Aristóteles", puede leerse como una introducción al capítulo II. En este primer capítulo se repasan los pasajes príncipes sobre los diversos significados de prioridad: Categorías 12 y Metafísica V 11 y se cruzan referencias con otros lugares del corpus. Se concluye que, a pesar de la diversidad de sentidos y aplicaciones de la noción de prioridad, existe una forma básica de prioridad: la ontológica. Vigo, en contra de la opinión de G.E.L. Owen y W. Leszl, desaprueba la supuesta dicotomía entre prioridad lógica y la ontológica, tema al que dedica el siguiente capítulo.

"Prioridad ontológica y prioridad lógica en la doctrina aristotélica de la sustancia" se enfoca en Metafísica VII 1. La noción de sustancia es crucial para entender los distintos tipos de prioridad. Los órdenes del ser, del lógos y del tiempo convergen en la sustancia. Se equivocan quienes atribuyen a Aristóteles una escisión entre prioridad lógica y prioridad ontológica. Los categoremas accidentales no pueden definirse de manera autónoma porque no existen sin la sustancia, mientras que la sustancia sí, pues ella existe autónomamente. Por tanto, lo ordinario en la teoría aristotélica es que prioridad lógica y prioridad ontológica coincidan.

Más complicada resulta la convergencia de la prioridad gnoseológica con la ontológica. El argumento de Vigo para defender tal convergencia se basa en la reducción de la oposición entre esencial y no esencial a la oposición

Diánoia, volumen LII, número 59 (noviembre 2007): pp. 199-204. 
entre modos de ser. Sólo podemos hablar de "lo no esencial" en la medida en que le atribuimos la esencia de modo derivado. Este sesgo del argumento, subsidiario de Metafísica IV, resulta especialmente afortunado. Sin la prioridad de la sustancia, todos los atributos se nivelan y pierden su jerarquía; nuestro acceso al objeto se desvanece en un haz de atributos más o menos inconexos.

El capítulo III, "Orden espacial y orden temporal según Aristóteles", se aboca al estudio del argumento de Física IV 11, 219a 10-21. Este pasaje pretende establecer que las propiedades continuidad del movimiento y sucesión temporal derivan de las propiedades correspondientes en la magnitud temporal. Dicha pretensión no exige un gran despliegue argumentativo en el caso del movimiento local. En este tipo de movimientos, los lapsos (tiempo) se corresponden fácilmente con segmentos (espacio). Los cambios de lugar se pueden describir en términos de sucesiones de "aquí y ahora". El asunto se complica cuando esta especie de reducción se aplica a otros tipos de movimiento, en especial a los cualitativos. La estrategia de Aristóteles, piensa Vigo, consiste en mostrar la imposibilidad de comprender las fases de un cambio cualitativo sin presuponer la referencia a objetos sustanciales situados en el espacio. La sustancia es sujeto real y lugar de todo proceso de cambio.

El capítulo IV, "El concepto de verdad teórica en Aristóteles", es particularmente interesante y, en algunos momentos, ligeramente provocativo. La tesis general es sencilla; la originalidad del texto está en los detalles. Siguiendo una larga tradición de comentadores, Vigo muestra que la estructura del enunciado predicativo S-P depende directamente de la ontología de la sustancia. Según el autor, este modelo adecuacionista requiere un concepto más primitivo de verdad, una verdad preproposicional. Metafísica IX 10 introduce un concepto de verdad que no cabe en el modelo S-P. Se trata de la verdad de lo simple, de aquello que no es compuesto y que, por ende, no puede remitir a la estructura S-P. El esquema de la verdad proposicional se corresponde con el enlace apofántico: la composición S-P. Este tipo de verdad pertenece a la katáphasis. Pero Aristóteles utiliza también las expresiones "tocar" (thigein) y "mencionar" (phánai) para el caso del empleo designativo de términos simples. Éste es el ámbito de la verdad preproposicional. Este uso suele remitirse al acto propio del nous (Ross), una especie de acceso privilegiado a la esencia de los objetos. No es ésta la interpretación de Vigo. Para él, al leer Metafísica IX 10 no se debe perder de vista que Aristóteles está hablando de la correcta aplicación de los términos simples al clasificar objetos. Cuando aplicamos con éxito uno de estos términos en un contexto designativo-referencial, basamos nuestra aplicación en un conocimiento vago e impreciso, y no en un conocimiento duro de la esencia de los objetos. Para identificar y utilizar correctamente el término $S$, no acudimos al modelo S-P. La verdad proposicional presupone el uso correcto de los términos S y P. Para acertar en la verdad de las proposiciones previamente hemos de usar correctamente la lista de las categorías. Vigo tira de este hilo y saca la consecuencia: "Al mismo tiempo que una teoría ontológica, la doctrina aristotélica de las categorías debe verse, en tal sentido, también, y con igual originalidad, como una teoría acerca de la posibilidad de la verdad, es

Diánoia, vol. LII, no. 59 (noviembre 2007). 
decir, como una doctrina de la verdad trascendental" (p. 53). Con esto, Vigo no pretende atribuir a Aristóteles una teoría naturalista de los nombres al estilo del Cratilo; lo que sí quiere señalar es que la posibilidad del lenguaje depende, entre otras cosas, de la consistencia en el empleo de los términos, una vez que se ha fijado su significado.

La tesis central del capítulo V - "Sustancia, sucesión y permanencia"- es que el enunciado de la forma S-P no se agota en la oposición lógica entre "sujeto" y "predicados", sino que remite a la oposición entre sustrato real del cambio y las determinaciones que le advienen durante su existencia temporal. El autor demuestra sobradamente esta hipótesis a partir del análisis de la caracterización formal de las sustancias de Categorías 5 y de la revisión de los principios del cambio de Física I, 7. Alrededor de este pasaje, Vigo explora el paralelismo entre los binomios permanencia-sucesión y sustancia-accidentes.

La parte más compleja de este capítulo es la revisión de Física IV 10-14, que se centra en el problema de la permanencia y la sucesión en el orden del tiempo; es decir, la aporía de la identidad y la alteridad del ahora: "El ahora es, en cierto sentido, el mismo (tò autó), y en cierto sentido no" (219b 12). La solución aristotélica supera la disyunción excluyente poniendo de manifiesto que identidad y alteridad son dos aspectos constitutivos del "ahora". El ahora es una propiedad del móvil y, por tanto, su identidad viene garantizada por la permanencia del sujeto.

El capítulo VI, "Aristóteles, Pseudo-Alejandro y la prioridad temporal de la sustancia", discute Metafísica VII 1, 1028b a 32-b2, donde se atribuye a la sustancia una prioridad ontológica, lógica, gnoseológica y temporal respecto de los accidentes. En un primer momento se sostiene que la sustancia es el significado primero y absoluto (haplos) de ser, porque ella es capaz de una existencia separada. En un segundo momento se atribuyen a la sustancia las tres restantes formas de prioridad. El Pseudo-Alejandro, comentando las líneas en cuestión, apela al criterio de separabilidad para argüir en favor de la prioridad temporal de la sustancia. Vigo analiza detenidamente el pasaje y pondera las dificultades textuales y sistemáticas para afirmar la prioridad temporal de la sustancia respecto del resto de las categorías, en los términos del Pseudo-Alejandro.

Un momento muy agudo en la argumentación de Vigo consiste en mostrar que el concepto intuitivo de prioridad temporal, digamos, de prioridad cronológica, es insuficiente para fundar la prioridad temporal de la sustancia. La mera sucesión en el tiempo no basta para distinguir categorialmente entre un sujeto y sus propiedades. Para construir un adecuado concepto de prioridad temporal ha de invocarse la oposición sucesión-permanencia. El Pseudo-Alejandro ve este punto, pero no lo explota. Vigo, en cambio, propone una lectura del concepto de prioridad en clave de permanencia. La sustancia es anterior a los accidentes porque es el sujeto que permanece a lo largo de los cambios.

"Homonimia, explicación y reducción en la Física", título del capítulo VII, continúa, a su modo, con el tema de la prioridad. Este apartado estudia algunas explicaciones reductivas de Física III y IV. Tales reducciones no consisten en la simple eliminación de las formas accidentales del ser. Son la focalización 
del significado desde el punto de vista de la sustancia: homonimia pròs hén o "significación focal", por utilizar la terminología de Owen. En Física, Aristóteles apunta a tratar las propiedades como dependientes de las sustancias. Éste es el espíritu de la "explicación reductiva".

El capítulo VIII evoca un comentario fenomenológico - no por ello falso o inexacto- del dictum aristotélico "el alma es, en cierto modo todas las cosas". El mundo de la experiencia no es como se nos ofrece sin la intervención activa del alma. La filosofía natural de Aristóteles no aspira a un ideal de objetividad en el que se prescinda completamente de la intervención activa del alma. En este modelo, la separación tajante entre lo que la modernidad ha denominado "sujeto" y "objeto" no tiene lugar propio. La Física de Aristóteles no es una teoría empírica de la naturaleza, sino una ontología del ser natural.

Esto no quiere decir, sin embargo, que Aristóteles asuma dogmáticamente el punto de partida de la "inmediatez". La conciencia del propio punto de partida es un indicio del espíritu crítico del Estagirita.

Los artículos no dejan cabos sueltos, a pesar de que no componen un tratado sistemático. Echo de menos, no por una falta de rigor en el texto, sino por un interés personal, una reflexión más amplia sobre el sustrato de la generación y la corrupción. Creo que tarde o temprano la prioridad de la sustancia requiere resolver el tema del sujeto de ese tipo de cambio, para poder garantizar la permanencia. En otras palabras, la prioridad de la sustancia remite a su permanencia, y ésta, me temo, a las complejas relaciones entre forma y materia primera.

La segunda parte de Estudios aristotélicos gira en torno a la teoría de la acción y destaca por su estupendo tratamiento del tema de la verdad práctica. El capítulo IX, "Razón práctica y tiempo", proporciona un lúcido comentario sobre la rehabilitación de la razón práctica. La modernidad tendió a reducir la razón a los términos de la teórica, que no es sino la inteligencia que contempla sub specie aeternitatis. Por contraposición, la razón práctica se construye a partir del horizonte de temporalidad. Lo propio del agente de praxis es su apertura al horizonte del futuro. En el razonamiento práctico se evalúa el aquí y el ahora con vistas a la vida concebida como una totalidad de sentido. Los agentes de praxis, por tanto, están siempre arrojados hacia el futuro: deciden sobre el hic et nunc con vistas a una precomprensión de sí mismos. Viven y comprenden el presente cara al futuro, a diferencia de los animales que se encuentran anclados en el presente.

A la luz de este esquema, deudor de Heidegger y Gadamer, Vigo explora los mecanismos de la motivación de la acción y del intelecto práctico. La razón práctica comprende el mundo en clave de medios y fines. El agente de praxis más diestro articula la trama de una manera armónica, conciliando el corto plazo con el mediano y el largo.

El conflicto de deseos es propio del agente de praxis y procede de su apertura al futuro. En los agentes anclados en el presente, el bien futuro no colisiona con el bien presente. Esta disyuntiva es exclusiva del ser humano. Pero el bien futuro siempre entraña un riesgo. La incertidumbre es un constitutivo de la

Diánoia, vol. LII, no. 59 (noviembre 2007). 
racionalidad práctica. Elegir en el presente con arreglo a un modelo de vida implica apostar por lo incierto, por lo contingente.

El capítulo X retoma el hilo del artículo precedente. La racionalidad práctica exige un modelo de verdad distinto del de las ciencias teóricas. Esto queda de manifiesto en el silogismo práctico, cuya conclusión no es una premisa, sino una acción. La verdad práctica no es, por tanto, una evaluación teórica sobre una acción determinada. No es proposición; es acierto efectivo. La confluencia del recto deseo del fin con el conocimiento eficaz de los medios pertinentes produce la verdad práctica.

Sin embargo, la noción de verdad práctica también tiene un componente adecuacionista. La acción acertada (o prácticamente verdadera) es aquella que procede de un deseo recto.

El capítulo XI discute el célebre pasaje de la Ética a Nicómaco VII sobre la akrasía, que ha despertado el interés de los estudiosos de la teoría de la acción. El encanto del análisis de Vigo consiste en resaltar que las reflexiones aristotélicas sobre la incontinencia se insertan en una teoría de la virtud, donde el énfasis está puesto en las disposiciones habituales del agente y no en las acciones.

El incontinente carece de los hábitos necesarios para ejecutar el ideal de vida que se ha propuesto; por ello queda anclado en el presente, disperso en la multiplicidad de estímulos sensibles del hic et nunc; no trasciende hacia el futuro. En consecuencia, el incontinente queda en deuda consigo mismo, fracasa en la realización de sus propias posibilidades; no ha logrado la unidad entre carácter y razón, porque sólo en la virtud se recupera esa unidad; gracias a la virtud se unifican razón y apetito.

El capítulo XII examina la conexión entre la verdad práctica y las virtudes téjnē y la phrónesis. Explora también las formas fundamentales de falsedad y error práctico. Vigo insiste en que, dado que las virtudes intelectuales son modos de estar en la verdad, no debe suponerse que los distintos hábitos intelectuales la poseen de la misma manera. El autor critica a quienes intentan reducir la verdad práctica al ámbito del lógos. Por el contrario, la verdad práctica es una verdad ontológica, una verdad de las cosas.

El capítulo XIII —que quizá quedaría mejor al final de la primera sección del libro- discute largamente con Cleary el tema de la prioridad ontológica y la unidad de la metafísica. J.J. Cleary (Aristotle on the Many Senses of Priority, 1988), no muy lejos de G. Patzig, traslada la relación pròs hén al plano vertical de las sustancias. Introduce cierto tipo de prioridad ontológica entre los diversos tipos de sustancia.

Sin embargo, la cuestión de la prioridad ontológica, advierte Vigo, no es lo más complejo: el meollo de la discusión se encuentra en la relación entre la prioridad lógica y la ontológica. ¿Cómo se relacionan ambas formas de prioridad en el orden vertical? Cleary tiende a admitir que lo usual en Aristóteles sería una separación entre ambas formas de prioridad, hipótesis que ya había sido difundida por Owen. Vigo no está de acuerdo con esta interpretación. Lo propio del proyecto ontológico de Aristóteles es la trabazón entre los tres órde- 
nes del ser, del lógos y del tiempo, una trabazón en la cual la sustancia aparece como centro básico de referencia y punto de convergencia de tales órdenes.

El capítulo XIV es un breve comentario (423-435) a la reconstrucción tetravalente de la asertórica aristotélica hecha por Niels Öffenberger. Vigo repasa las líneas maestras del proyecto y muestra cierto escepticismo sobre su alcance. El modelo tetravalente no captura la distinción entre verdades absolutas y verdades generales, tan importante en la epistemología aristotélica. La física sublunar sólo tiene validez para la gran mayoría de los casos (hos epí tò polú) y la lógica tetravalente no captura este matiz.

El libro de Vigo es valioso por muchas razones. Un primer motivo, que no es menor, es que su autor pasa revista a la bibliografía especializada en español, francés, alemán e inglés. Una costumbre que, lamentablemente, se está perdiendo entre ciertos especialistas proclives a prescindir de las magníficas obras publicadas en alemán y francés. En este mismo terreno de la bibliografía, el autor demuestra su familiaridad con los comentarios clásicos y medievales del corpus, combinación que no resulta tan frecuente como podría esperarse.

Un tercer aspecto que destacar es la claridad a la hora de analizar textos y de hilvanar argumentos. El estilo conciso de Vigo va más allá del mero análisis filológico y plantea problemas teóricos. El volumen es, pues, un texto sugerente y sólido.

HÉCTOR ZAGAL

Facultad de Filosofía

Universidad Panamericana

hzagal@mx.up.mx

Al-Aš'arī, Contra heterodoxos (al-Luma') o lo que deben creer los musulmanes, traducción de Carlos A. Segovia, Biblioteca Nueva, Madrid, 2006, 253 pp.

La filosofía (falsafa) considerada como disciplina autónoma y estrictamente racional fue, en el contexto árabe-musulmán del medioevo, un saber heredado de los helénicos. Antes del siglo viII, para los musulmanes existían solamente dos disciplinas derivadas de la religión: la teología (kalām) y la jurisprudencia (fiqh). Ambas fungieron y fungen como herramientas para comprender la revelación coránica y establecer algunos criterios de acción que no son del todo claros en el Libro. Las escuelas jurídico-teológicas se diversificaron rápidamente, de modo que para el siglo VIII había ya sectores religiosos bien integrados que discutían la ley contenida en el Libro. Algunos de esos sectores vieron con suspicacia la ciencia filosófica. El grupo religioso denominado mu'tazilí fue el primero en abrirse a la filosofía y en romper con la interpretación literal del Corán admitiendo el uso de los argumentos dialécticos para su labor exegética.

Diánoia, volumen LII, número 59 (noviembre 2007): pp. 204-209. 